\title{
Porting an Open Information Extraction System from English to German
}

\author{
Tobias Falke $^{\dagger} \quad$ Gabriel Stanovsky $^{\ddagger} \quad$ Iryna Gurevych $^{\dagger} \quad$ Ido Dagan $^{\ddagger}$ \\ ${ }^{\dagger}$ Research Training Group AIPHES and UKP Lab \\ Computer Science Department, Technische Universität Darmstadt

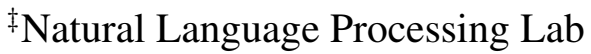 \\ Department of Computer Science, Bar-Ilan University
}

\begin{abstract}
Many downstream NLP tasks can benefit from Open Information Extraction (Open IE) as a semantic representation. While Open IE systems are available for English, many other languages lack such tools. In this paper, we present a straightforward approach for adapting PropS, a rule-based predicate-argument analysis for English, to a new language, German. With this approach, we quickly obtain an Open IE system for German covering $89 \%$ of the English rule set. It yields $1.6 \mathrm{n}$-ary extractions per sentence at $60 \%$ precision, making it comparable to systems for English and readily usable in downstream applications. ${ }^{1}$
\end{abstract}

\section{Introduction}

The goal of Open Information Extraction (Open IE) is to extract coherent propositions from a sentence, each represented as a tuple of a relation phrase and one or more argument phrases (e.g., born in (Barack Obama; Hawaii)). Open IE has been shown to be useful for a wide range of semantic tasks, including question answering (Fader et al., 2014), summarization (Christensen et al., 2013) and text comprehension (Stanovsky et al., 2015), and has consequently drawn consistent attention over the last years (Banko et al., 2007; Wu and Weld, 2010; Fader et al., 2011; Akbik and Löser, 2012; Mausam et al., 2012; Del Corro and Gemulla, 2013; Angeli et al., 2015).

Although similar applications of Open IE in other languages are obvious, most previous work focused

\footnotetext{
${ }^{1}$ Source code and online demo available at https: //github.com/UKPLab/props-de
}

on English, with only a few recent exceptions (Zhila and Gelbukh, 2013; Gamallo and Garcia, 2015). For most languages, Open IE systems are still missing. While one could create them from scratch, as it was done for Spanish, this can be a very laborious process, as state-of-the-art systems make use of handcrafted, linguistically motivated rules. Instead, an alternative approach is to transfer the rule sets of available systems for English to the new language.

In this paper, we study whether an existing set of rules to extract Open IE tuples from English dependency parses can be ported to another language. We use German, a relatively close language, and the PropS system (Stanovsky et al., 2016) as examples in our analysis. Instead of creating rule sets from scratch, such a transfer approach would simplify the rule creation, making it possible to build Open IE systems for other languages with relatively low effort in a short amount of time. However, challenges we need to address are differences in syntax, dissimilarities in the corresponding dependency representations as well as language-specific phenomena. Therefore, the existing rules cannot be directly mapped to the German part-of-speech and dependency tags in a fully automatic way, but require a careful analysis as carried out in this work. Similar manual approaches to transfer rule-based systems to new languages were shown to be successful, e.g. for temporal tagging (Moriceau and Tannier, 2014), whereas fully automatic approaches led to less competitive systems (Strötgen and Gertz, 2015).

Our analysis reveals that a large fraction of the PropS rule set can be easily ported to German, requiring only small adaptations. With roughly $10 \%$ 
Sehenswert sind die Orte San Jose und San Andres, die an der nördlichen Küste des Petén-Itzá-Sees liegen.

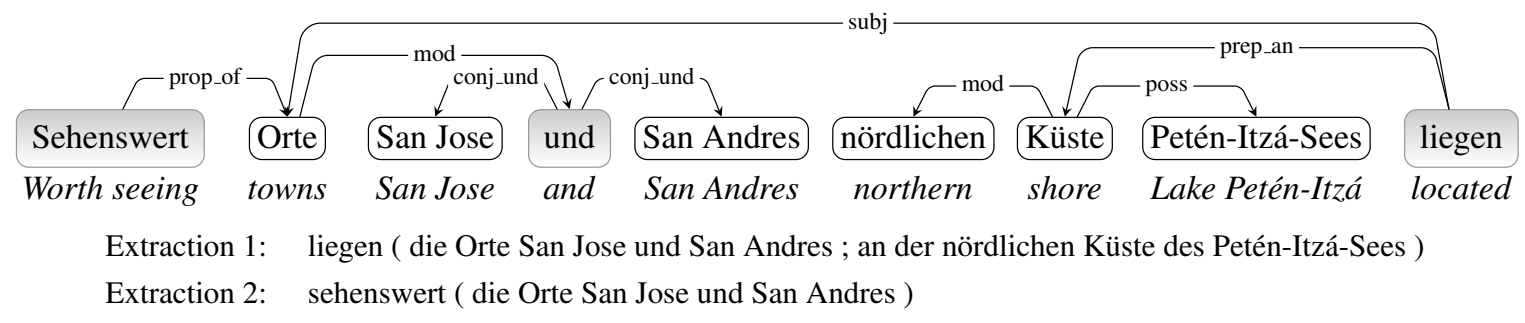

Figure 1: PropS representation for Worth seeing are the towns San Jose and San Andres, which are located on the northern shore of Lake Petén-Itzá. Grey boxes indicate predicates. Two Open IE tuples, one unary and one binary, are extracted from this sentence.

of the effort that went into the English system, we could build a system for German covering $89 \%$ of the rule set. As a result, we present PropsDE, the first Open IE system for German. In an intrinsic evaluation, we show that its performance is comparable with systems for English, yielding 1.6 extractions per sentence with an overall precision of $60 \%$.

\section{Background}

Open Information Extraction Open IE was introduced as an open variant of traditional Information Extraction (Banko et al., 2007). Since its inception, several extractors were developed. The majority of them, namely ReVerb (Fader et al., 2011), KrakeN (Akbik and Löser, 2012), Exemplar (Mesquita et al., 2013) and ClausIE (Del Corro and Gemulla, 2013), successfully used rule-based strategies to extract tuples. Alternative approaches are variants of self-supervision, as in TextRunner (Banko et al., 2007), WOE (Wu and Weld, 2010) and OLLIE (Mausam et al., 2012), and semanticallyoriented approaches utilizing semantic role labeling (Open IE-4 ${ }^{2}$ ) or natural logic (Angeli et al., 2015). While TextRunner and ReVerb require only POS tagging as preprocessing to allow a high extraction speed, the other systems rely on dependency parsing to improve the extraction precision.

For non-English Open IE, ExtrHech has been presented for Spanish (Zhila and Gelbukh, 2013). Similar as the English systems, it uses a set of extraction rules, specifically designed for Spanish in this case. More recently, ArgOE (Gamallo and Garcia, 2015) was introduced. It manages to extract tuples in several languages with the same rule set, relying on a

\footnotetext{
${ }^{2}$ https://github.com/knowitall/openie
}

dependency parser that uses a common tagset for five European languages. However, an evaluation for English and Spanish revealed that this approach cannot compete with the systems specifically built for those languages. To the best of our knowledge, no work on Open IE for German exists.

Open IE with PropS Stanovsky et al. (2016) recently introduced PropS, a rule-based converter turning dependency graphs for English into typed graphs of predicates and arguments. An example is shown in Figure 1 (in German). Compared to a dependency graph, the representation masks non-core syntactic details, such as tense or determiners, unifies semantically equivalent constructions, such as active/passive, and explicates implicit propositions, such as indicated by possessives or appositions.

The resulting graph can be used to extract Open IE tuples in a straightforward way. Every nonnested predicate node pred in the graph, together with its $n$ argument-subgraphs $\arg g_{i}$, yields a tuple $\operatorname{pred}\left(\arg _{1} ; \ldots ; \arg _{n}\right)$. With this approach, PropS is most similar to KrakeN and ClausIE, applying rules to a dependency parse. However, due to additional nodes for implicit predicates, it can also make extractions that go beyond the scope of other systems, such as has ( Michael; bicycle) from Michael's bicycle is red. In line with more recent Open IE systems, this strategy extracts tuples that are not necessarily binary, but can be unary or of higher arity.

\section{Analysis of Portability}

Approach For each rule of the converter that transforms a dependency graph to the PropS graph, we assess its applicability for German. A rule is applied to a part of the graph if certain conditions are 
fulfilled, expressed using dependency types, POS tags and lemmas. As we already pointed out in the introduction, several differences between the dependency and part-of-speech representations for English and German make a fully automatic translation of these rules impossible. We therefore manually analyzed the portability of each rule and report the findings in the next section.

While using Universal Dependencies (Nivre et al., 2016) could potentially simplify porting the rules, we chose not to investigate this option due to the ongoing nature of the project and focused on the established representations for now. In line with the English system, that works on collapsed Stanford dependencies (de Marneffe and Manning, 2008), we assume a similar input representation for German that can be obtained with a set of collapsing and propagation rules provided by Ruppert et al. (2015) for TIGER dependencies (Seeker and Kuhn, 2012).

Findings Overall, we find that most rules can be used for German, mainly because syntactic differences, such as freer word order (Kübler, 2008), are already masked by the dependency representation (Seeker and Kuhn, 2012). About $\mathbf{3 8 \%}$ of the rule set can be directly ported to German, solely replacing dependency types, POS tags and lemmas with their German equivalents. As an example, the rule removing negation tokens looks for neg dependencies in the graph, for which a corresponding type $N G$ exists in German. We found similar correspondences to remove punctuation and merge proper noun and number compounds. In addition, we can also handle appositions and existentials with direct mappings.

For $35 \%$ of the English rules, small changes are necessary, mainly because no direct mapping to the German tag set is possible or the annotation style differs. For instance, while English has a specific type det to link determiners to their governor, a more generic type $(N K)$ is used in German. Instead, determiners can be detected by part-of-speech:

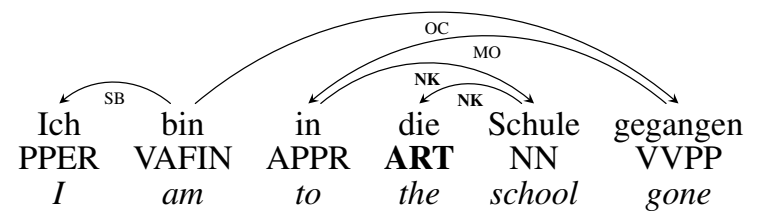

Another type of difference exists with regard to the representation of auxiliary verb constructions. In
Stanford dependencies, main verbs govern all auxiliaries, whereas in TIGER dependencies, an auxiliary heads the main verb. The above example shows this for gone and am. Therefore, all rules identifying and removing auxiliaries and modals have to be adapted to account for this difference.

With similar changes as discussed for determiners, we can also handle possessive and copular constructions. The graph for Michael's bicycle is red, for example, features an additional predicate have to explicate the implicit possessive relation, while red becomes an adjectival predicate, omitting is:

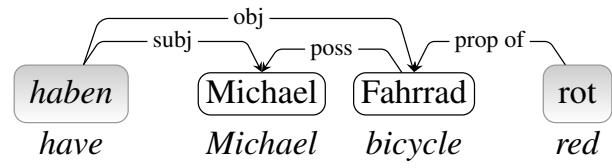

Moreover, conditional constructions can be processed with slight changes as well. Missing a counterpart for the type mark, we instead look for subordinating conjunctions by part-of-speech. In fact, we found conditionals to be represented more consistently across different conjunctions, making their handling in German easier than in English.

More substantial changes are necessary for the remaining $\mathbf{2 7 \%}$ of the rules. To represent active and passive in a uniform way, in passive clauses, PropS turns the subject into an object and a potential byclause into the subject. For English, these cases are indicated by the presence of passive dependencies such as nsubjpass. For German, however, no counterparts exist. As an alternative strategy, we instead look for past participle verbs (by POS tag) that are governed by a form of the auxiliary werden (Schäfer, 2015). Instances of the German static passive (Zustandspassiv) are, in contrast, handled like copulas. Another deviation from the English system is necessary for relative clauses. PropS heavily relies on the Stanford dependency converter, which propagates dependencies of the relative pronoun to its referent. The German collapser does not have this feature, and we therefore implement it as an additional transformation (see subj(liegen;Orte) in Figure 1).

To abstract away from different tenses, PropS represents predicates with their lemma, indicating the original tense as a feature, as detected with a set of rules operating on POS tags. For German, no tense information is contained in POS tags, but instead, a morphological analysis can provide it. Determining 
the overall tense of a sentence based on that requires a new set of rules, as the grammatical construction of tenses differs between German and English. PropS also tries to heuristically identify raising constructions, in which syntactic and semantic roles of arguments differ. In German, this phenomenon occurs in similar situations, such as in Michael scheint zu lächeln (Michael seems to smile), in which Michael is not the semantic subject of scheinen, though syntactically it is. To determine these cases heuristically, an empirically derived list of common raising verbs, such as done by Chrupała and van Genabith (2007) for English, needs to be created.

An additional step that is necessary during the lemmatization of verbs for German is to recover separated particles. For example, a verb like ankommen (arrive) can be split in a sentence such as $\mathrm{Er}$ kam an (He arrived), moving the particle to the end of the sentence, with a potentially large number of other tokens in between. We can reliably reattach these particles based on the dependency parse. Another addition to the rules that we consider important is to detect subjunctive forms of verbs and indicate the mood with a specific feature for the predicate. A morphological analysis provides the necessary input. Compared to English, the usage of the subjunctive is much more common, usually to indicate either unreality or indirect speech (Thieroff, 2004).

\section{German Open IE System}

Following our analysis, we implemented a German version of PropS, named PropsDE. It uses matetools for POS tagging, lemmatizing and parsing (Bohnet et al., 2013). Dependencies are collapsed and propagated with JoBimText (Ruppert et al., 2015). The rule set covers $89 \%$ of the English rules, lacking only the handling of raising-to-subject verbs and more advanced strategies for coordination constructions and tense detection. To assign confidence scores, PropsDE uses a logistic regression model trained to predict the correctness of extractions. Figure 1 illustrates some extracted tuples. Based on correspondence with the authors of the English system, we conclude that we were able to implement the German version with roughly $10 \%$ of the effort they reported. This shows that our approach of manually porting a rule-based system can overcome the lack of a tool for another language with reasonable effort in a short amount of time.

\section{Experiments}

Experimental Setup Following the common evaluation protocol for Open IE systems, we manually label extractions made by our system. For this purpose, we created a new dataset consisting of 300 German sentences, randomly sampled from three sources of different genres: news articles from TIGER (Brants et al., 2004), German web pages from CommonCrawl (Habernal et al., 2016) and featured Wikipedia articles. For the treebank part, we ran our system using both gold and parsed dependencies to analyze the impact of parsing errors.

Every tuple extracted from this set of 300 sentences was labeled independently by two annotators as correct or incorrect. In line with previous work, they were instructed to label an extraction as incorrect if it has a wrong predicate or argument, including overspecified and incomplete arguments, or if it is well-formed but not entailed by the sentence. Unresolved co-references were not marked as incorrect. We observed an inter-annotator agreement of $85 \%(\kappa=0.63)$. For the evaluation, we merged the labels, considering an extraction as correct only if both annotators labeled it as such. Results are measured in terms of precision, the fraction of correct extractions, and yield, the total number of extractions. A precision-yield curve is obtained by decreasing a confidence threshold. The confidence predictor was trained on a separate development set.

Results From the whole corpus of 300 sentences, PropsDE extracted 487 tuples, yielding on average 1.6 per sentence with 2.9 arguments. $60 \%$ of them were labeled as correct. Table 1 shows that most extractions are made from Wikipedia articles, whereas the highest precision can be observed for newswire text. According to our expectations, web pages are most challenging, presumably due to noisier language. These differences between the genres can also be seen in the precision-yield curve (Figure 2).

For English, state-of-the-art systems show a similar performance. In a direct comparison of several systems carried out by Del Corro and Gemulla (2013), they observed overall precisions of 58\% (Reverb), 57\% (ClausIE), 43\% (WOE) and 43\% 


\begin{tabular}{l|cc|cc} 
Genre & Sentences & Length & Yield & Precision \\
\hline News* & 100 & 19.3 & 142 & 78.9 \\
\hline News & 100 & 19.3 & 144 & 70.8 \\
Wiki & 100 & 21.4 & 178 & 61.8 \\
Web & 100 & 19.2 & 165 & 49.1 \\
\hline Total & 300 & 20.0 & 487 & 60.2
\end{tabular}

Table 1: Corpus size (length in token) and system performance by genre. News* used gold trees and is not included in total.

(OLLIE) on datasets of similar genre. The reported yield per sentence is higher for ClausIE (4.2), OLLIE (2.6) and WOE (2.1), but smaller for Reverb (1.4). However, we note that in their evaluation, they configured all systems to output only two-argumentextractions. For example, from a sentence such as

The principal opposition parties boycotted the polls after accusations of vote-rigging.

OLLIE can either make two binary extractions

boycotted ( the principal opposition parties ; the polls )

boycotted the polls after (the principal opposition parties ; accusations of vote-rigging )

or just a single extraction with three arguments. PropS always extracts the combined tuple

boycotted ( the principal opposition parties, the polls, after accusations of vote-rigging ),

which is in line with the default configuration of more recent Open IE systems.

For the sake of comparability, we conjecture that the yield of our system would increase if we broke down higher-arity tuples in a similar fashion: Assuming that every extraction with $n$ arguments, $n>$ 2 , can be split into $n-1$ separate extractions, our system's yield would increase from 1.6 to 3.0. That is in line with the numbers reported above for the binary configuration for English. Overall, this indicates a reasonable performance of our straightforward porting of PropS to German.

Extractions were most frequently labeled as incorrect due to false relation labels (32\%), overspecified arguments (21\%) and wrong word order in arguments (19\%). Analyzing our system's performance on the treebank, we can see that the usage of gold dependencies increases the precision by 8 percentage

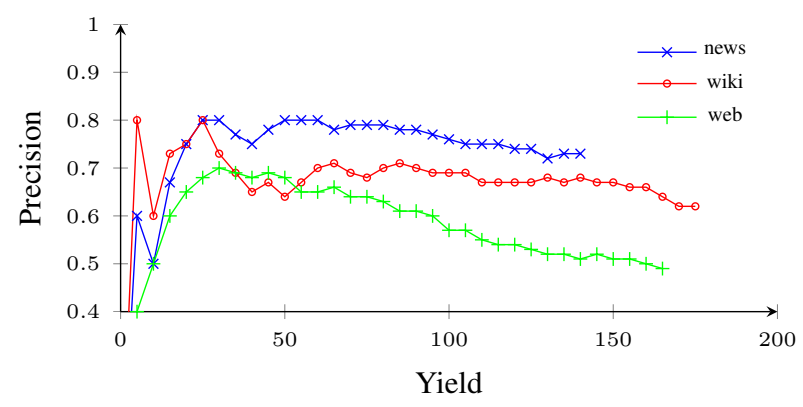

Figure 2: Extraction precision at increasing yield by genre.

points, making parsing errors responsible for about $28 \%$ of the incorrect extractions. Since the matetools parser is trained on the full TIGER treebank, including our experimental data, its error contribution on unseen data might be even higher.

\section{Conclusion}

Using PropS and German as examples, we showed that a rule-based Open IE system for English can be ported to another language in a reasonable amount of time. As a result, we presented the first Open IE system for German. In the future, studies targeting less similar languages could further evaluate the portability of PropS. Directions for future work on PropsDE are extensions of the rule set to better cover complex coordination constructions, nested sentences and nominal predicates.

\section{Acknowledgments}

This work has been supported by the DFG-funded research training group "Adaptive Preparation of Information from Heterogeneous Sources" (AIPHES, GRK 1994/1), by the German Research Foundation through the German-Israeli Project Cooperation (DIP, grant DA 1600/1-1 and grant GU 798/17-1) and by the Volkswagen Foundation as part of the Lichtenberg-Professorship Program under grant No. I/82806.

\section{References}

Alan Akbik and Alexander Löser. 2012. KrakeN: Nary Facts in Open Information Extraction. In Proceedings of the Joint Workshop on Automatic Knowledge Base Construction \& Web-scale Knowledge Extraction, pages 52-56, Montreal, Canada. 
Gabor Angeli, Melvin Johnson Premkumar, and Christopher D. Manning. 2015. Leveraging Linguistic Structure For Open Domain Information Extraction. In Proceedings of the 53rd Annual Meeting of the Association for Computational Linguistics and the 7th International Joint Conference on Natural Language Processing, pages 344-354, Beijing, China.

Michele Banko, Michael J. Cafarella, Stephen Soderland, Matt Broadhead, and Oren Etzioni. 2007. Open Information Extraction from the Web. In Proceedings of the 20th International Joint Conference on Artifical Intelligence, pages 2670-2676, Hyderabad, India.

Bernd Bohnet, Joakim Nivre, Igor Boguslavsky, Richárd Farkas, Filip Ginter, and Jan Hajič. 2013. Joint Morphological and Syntactic Analysis for Richly Inflected Languages. Transactions of the Association for Computational Linguistics, 1(0):415-428.

Sabine Brants, Stefanie Dipper, Peter Eisenberg, Silvia Hansen-Schirra, Esther König, Wolfgang Lezius, Christian Rohrer, George Smith, and Hans Uszkoreit. 2004. TIGER: Linguistic Interpretation of a German Corpus. Research on Language and Computation, 2(4):597-620.

Janara Christensen, Mausam, Stephen Soderland, and Oren Etzioni. 2013. Towards Coherent MultiDocument Summarization. In Proceedings of the 2013 Conference of the North American Chapter of the Association for Computational Linguistics: Human Language Technologies, pages 1163-1173, Atlanta, GA, USA.

Grzegorz Chrupała and Josef van Genabith. 2007. Using Very Large Corpora to Detect Raising and Control Verbs. In Proceedings of the Lexical Functional Grammar 2007 Conference, pages 597-620, Stanford, CA, USA.

Marie-Catherine de Marneffe and Christopher D. Manning. 2008. The Stanford typed dependencies representation. In Proceedings of the 22nd International Conference on Computational Linguistics, pages 1-8, Manchester, United Kingdom.

Luciano Del Corro and Rainer Gemulla. 2013. ClausIE: Clause-Based Open Information Extraction. In Proceedings of the 22nd International Conference on the World Wide Web, pages 355-366, Rio de Janeiro, Brazil.

Anthony Fader, Stephen Soderland, and Oren Etzioni. 2011. Identifying Relations for Open Information Extraction. In Proceedings of the Conference on Empirical Methods in Natural Language Processing, pages 1535-1545, Edinburgh, United Kingdom.

Anthony Fader, Luke Zettlemoyer, and Oren Etzioni. 2014. Open question answering over curated and extracted knowledge bases. In Proceedings of the 20th
ACM SIGKDD International Conference on Knowledge Discovery and Data Mining, pages 1156-1165, New York, NY, USA.

Pablo Gamallo and Marcos Garcia. 2015. Multilingual Open Information Extraction. In Proceedings of the 17th Portuguese Conference on Artificial Intelligence, volume 9273 of Lecture Notes in Computer Science, pages 711-722, Coimbra, Portugal.

Ivan Habernal, Omnia Zayed, and Iryna Gurevych. 2016. C4Corpus: Multilingual Web-size corpus with free license. In Proceedings of the 10th International Conference on Language Resources and Evaluation, pages 914-922, Portorož, Slovenia.

Sandra Kübler. 2008. The PaGe 2008 shared task on parsing German. In Proceedings of the ACL-08: HLT Workshop on Parsing German (PaGe-08), pages 5563, Columbus, OH, USA.

Mausam, Michael Schmitz, Robert Bart, Stephen Soderland, and Oren Etzioni. 2012. Open Language Learning for Information Extraction. In Proceedings of the 2012 Joint Conference on Empirical Methods in Natural Language Processing and Computational Natural Language Learning, pages 523-534, Jeju Island, Korea.

Filipe Mesquita, Jordan Schmidek, and Denilson Barbosa. 2013. Effectiveness and Efficiency of Open Relation Extraction. In Proceedings of the 2013 Conference on Empirical Methods in Natural Language Processing, pages 447-457, Seattle, WA, USA.

Véronique Moriceau and Xavier Tannier. 2014. French Resources for Extraction and Normalization of Temporal Expressions with HeidelTime. In Proceedings of the Ninth International Conference on Language Resources and Evaluation (LREC'14), pages 3239-3243, Reykjavik, Iceland.

Joakim Nivre, Marie-Catherine de Marneffe, Filip Ginter, Yoav Goldberg, Jan Hajič, Christopher D. Manning, Ryan McDonald, Slav Petrov, Sampo Pyysalo, Natalia Silveira, Reut Tsarfaty, and Daniel Zeman. 2016. Universal Dependencies v1: A Multilingual Treebank Collection. In Proceedings of the 10th International Conference on Language Resources and Evaluation, pages 1659-1666, Portorož, Slovenia.

Eugen Ruppert, Jonas Klesy, Martin Riedl, and Chris Biemann. 2015. Rule-based Dependency Parse Collapsing and Propagation for German and English. In Proceedings of the GSCL 2015, pages 58-66, Duisburg, Germany.

Roland Schäfer. 2015. Einführung in die grammatische Beschreibung des Deutschen. Language Science Press, Berlin, Germany.

Wolfgang Seeker and Jonas Kuhn. 2012. Making Ellipses Explicit in Dependency Conversion for a Ger- 
man Treebank. In Proceedings of the Eighth International Conference on Language Resources and Evaluation, pages 3132-3139, Istanbul, Turkey.

Gabriel Stanovsky, Ido Dagan, and Mausam. 2015. Open IE as an Intermediate Structure for Semantic Tasks. In Proceedings of the 53rd Annual Meeting of the Association for Computational Linguistics and the 7 th International Joint Conference on Natural Language Processing, pages 303-308, Beijing, China.

Gabriel Stanovsky, Jessica Ficler, Ido Dagan, and Yoav Goldberg. 2016. Getting More Out Of Syntax with PropS. arXiv:1603.01648.

Jannik Strötgen and Michael Gertz. 2015. A Baseline Temporal Tagger for all Languages. In Proceedings of the 2015 Conference on Empirical Methods in Natural Language Processing, pages 541-547, Lisbon, Portugal.

Rolf Thieroff. 2004. The subjunctive mood in German and in the Germanic languages. In Focus on Germanic Topology, pages 315-358. Akademie Verlag, Berlin, Germany.

Fei Wu and Daniel S. Weld. 2010. Open Information Extraction Using Wikipedia. In Proceedings of the 48th Annual Meeting of the Association for Computational Linguistics, pages 118-127, Uppsala, Sweden.

Alisa Zhila and Alexander Gelbukh. 2013. Comparison of open information extraction for English and Spanish. In Proceedings of the International Conference on Computational Linguistics and Intellectual Technologies (Dialogue 2013), pages 714-722, Bekasovo, Russia. 